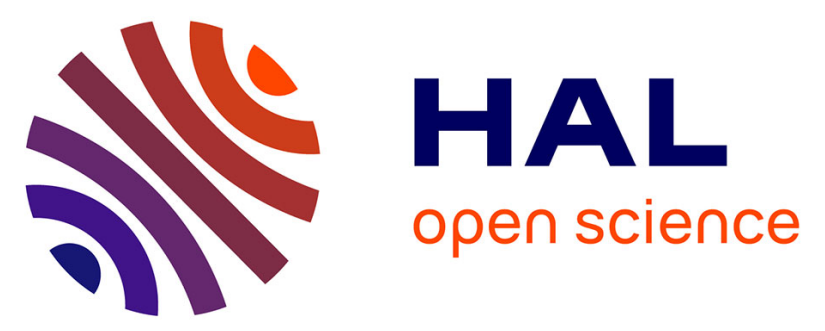

\title{
Hierarchical Structure of NiMo Hydrodesulfurization Catalysts Determined by Ptychographic X-ray Computed Tomography
}

Johannes Ihli, Leonid Bloch, Frank Krumeich, Klaus Wakonig, Mirko Holler, Manuel Guizar-Sicairos, Thomas Weber, Julio Cesar da Silva, Jeroen Anton van Bokhoven

\section{To cite this version:}

Johannes Ihli, Leonid Bloch, Frank Krumeich, Klaus Wakonig, Mirko Holler, et al.. Hierarchical Structure of NiMo Hydrodesulfurization Catalysts Determined by Ptychographic X-ray Computed Tomography. Angewandte Chemie International Edition, 2020, 59 (39), pp.17266-17271. 10.1002/anie.202008030 . hal-02900805

\section{HAL Id: hal-02900805 \\ https://hal.science/hal-02900805}

Submitted on 24 Jul 2020

HAL is a multi-disciplinary open access archive for the deposit and dissemination of scientific research documents, whether they are published or not. The documents may come from teaching and research institutions in France or abroad, or from public or private research centers.
L'archive ouverte pluridisciplinaire HAL, est destinée au dépôt et à la diffusion de documents scientifiques de niveau recherche, publiés ou non, émanant des établissements d'enseignement et de recherche français ou étrangers, des laboratoires publics ou privés. 


\section{Hierarchical Structure of NiMo Hydrodesulfurization Catalysts Determined by Ptychographic X-ray Computed Tomography}

Johannes Ihli*, Leonid Bloch, Frank Krumeich, Klaus Wakonig, Mirko Holler, Manuel Guizar-Sicairos, Thomas Weber, Julio Cesar da Silva, Jeroen Anton van Bokhoven*

Dr Johannes Ihli*, Mr Klaus Wakonig, Dr Mirko Holler, Dr Manuel Guizar-Sicairos, Prof Jeroen Anton van Bokhoven

Paul Scherrer Institut, 5232 Villigen PSI, CH

Mr Leonid Bloch, Dr Frank Krumeich, Prof Jeroen Anton van Bokhoven* Inst. f. Chemie- u. Bioingenieurwissenschaften, $\mathrm{ETH}_{\text {Zürich }}, 8092$ Zürich, $\mathrm{CH}$

Mr Leonid Bloch and Dr Julio Cesar da Silva

European Synchrotron Radiation Facility, 38000 Grenoble, FR

Mr Klaus Wakonig

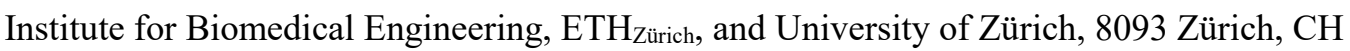

Prof Thomas Weber

Shell Technology Centre Amsterdam, 1031HW Amsterdam, NL

Dr Julio Cesar da Silva

Institut Néel CNRS UPR2940, 38042 Grenoble, FR

* Correspondence and requests for materials should be addressed to

jeroen.vanbokhoven@chem.ethz.ch or johannes.ihli@psi.ch 


\begin{abstract}
:
Hydrodesulphurization, the removal of sulphur from crude oils, is an essential catalytic process in the petroleum industry safeguarding the production of clean hydrocarbons. Sulphur removal is critical for the functionality of downstream processes and vital to the elimination of environmental pollutants. The effectiveness of such an endeavour is among other factors determined by the structural arrangement of the heterogeneous catalyst. Namely, the accessibility of the catalytically active molybdenum disulphide $\left(\mathrm{MoS}_{2}\right)$ slabs located on the surfaces of a porous alumina carrier. Here, we examined a series of pristine sulfided Mo and NiMo hydrodesulphurization catalysts of increasing metal loading prepared on commercial alumina carriers using ptychographic X-ray computed nanotomography. Structural analysis revealed a build consisting of two interwoven support matrix elements differing in nanoporosity. With increasing metal loading, approaching that of industrial catalysts, these matrix elements exhibit a progressively dissimilar $\mathrm{MoS}_{2}$ surface coverage as well as $\mathrm{MoS}_{2}$ cluster formation at the matrix element boundaries. This is suggestive of metal deposition limitations and/ or catalyst activation and following prohibitive of optimal catalytic utilization. These results will allow for diffusivity calculations, a better rationale of current generation catalyst performance as well as a better distribution of the active phase in next-generation hydrodesulphurization catalysts.
\end{abstract}


Hydrodesulphurization (HDS), the catalytic process of removing harmful sulphur from crude oils, is an essential process in the petroleum industry. ${ }^{[1]}$ More than 2 billion tons of crude oil are the subject of hydrodesulphurization processes annually. ${ }^{[2]}$ Sulphur removal guarantees the production of clean hydrocarbons, which in turn facilitate the functionality of downstream processes and reduce the emission of environmental pollutants. In view of our increasing reliance on heavier feedstocks and stricter environmental regulations, associated with a continuing consumption of petroleum-based fuels, more effective and environmentally stable catalysts need to be developed. ${ }^{[1 \mathrm{a}, 3]}$

Industrial HDS catalysts consist of a porous and hierarchically structured alumina $\left(\gamma-\mathrm{Al}_{2} \mathrm{O}_{3}\right)$ carrier that supports numerous catalytically active NiMoS or CoMoS nanoparticles. ${ }^{[1 c]}$ The alumina carrier is usually prepared from an aqueous dispersion of boehmite $(\gamma-\mathrm{AlO}(\mathrm{OH}))$ nanoparticles and obtained after calcination at temperatures greater than $550^{\circ} \mathrm{C}$. ${ }^{[4]}$ The often pellet-shaped alumina carrier is then impregnated with aqueous solutions containing molybdenum and cobalt or nickel, resulting in the formation of oxidic precursors on the surface of the carrier following drying and calcination. ${ }^{[2 b, 4]}$ Finally, and prior to their use as catalysts, these oxidic precursors are activated through sulfidation. Said activation is achieved either through gas-phase or liquid-phase sulphidation at temperatures of roughly $350^{\circ} \mathrm{C}$, yielding molybdenum disulphide $\left(\mathrm{MoS}_{2}\right)$ type particles or slabs. ${ }^{[5]}$

These particles are on average smaller than $10 \mathrm{~nm}$ in diameter and may contain $\mathrm{Co}$ or $\mathrm{Ni}$ atoms at the particle edge positions. The latter are considered to be the primary active sites in the catalytic desulfurization reaction. ${ }^{[6]}$ During this reaction, sulphur-containing molecules present in crude oils are decomposed on the catalyst surface into hydrocarbons and dihydrogen sulphide at hydrogen pressures between 40 and 80 bar and a temperature of around $350^{\circ} \mathrm{C}^{[1 \mathrm{c}, 7]}$

Considering the advanced understanding of the reaction mechanism and the nature of the active sites in model reactions, ${ }^{[1 \mathrm{c}, 6 \mathrm{~b}, 8]}$ the obvious area of catalyst improvement lies within an improved utilisation and dispersion of the metal sulphide particles on the porous support matrix. Specifically, increasing the diffusion/access of larger molecules to the catalytically active molybdenum disulphide slabs is of utmost interest. Yet, a three-dimensional characterisation of common industrial HDS catalysts on the support or carrier level, i.e. macro and mesopore level, facilitating such an improvement is largely amiss.

In this study, we introduce ptychographic X-ray computed nanotomography (PXCT) ${ }^{[9]}$ to characterize the structural make-up of industrial-like HDS catalysts. PXCT is a 
nanotomography technique that provides quantitative tomograms of electron density when acquired away from sample-relevant X-ray absorption edges. The following results and discussion will focus on the structure of a commercial high-surface-area alumina carrier, and two pristine catalysts with increasing metal loadings prepared on this carrier.

Evident across all samples is a catalyst build consisting of two interwoven support matrix elements differing in nanoporosity that are connected by larger diffusion pathways which undergo subtle changes upon metal loading or molybdenum disulphide formation. With increasing metal loading these matrix elements exhibit progressively dissimilar $\mathrm{MoS}_{2}$ coverage as well as $\mathrm{MoS}_{2}$ cluster formation at the matrix element boundaries. This is suggestive of metal deposition limitations and following prohibitive of optimal catalytic utilization. Results will allow for diffusivity calculations in current generation as well as a better distribution of the active phase in next-generation hydrodesulphurization catalysts.

Pristine HDS catalysts in the form of centimetre-sized catalyst pellets were provided by the Shell Oil Company. Examined were pellets either entirely consisting of the (i) bare alumina support, (ii) a support loaded with 5 wt. \% Mo or (iii) loaded with 10 wt. \% Mo and 2.3 wt. \% Ni. The latter two following catalyst activation or $\mathrm{MoS}_{2}$ formation. Using lasermicrodissection, we isolated roughly shaped cylinders, $<20 \mu \mathrm{m}$ in diameter, from the centre of each of the provided catalyst pellets. These cylinders were then analysed using PXCT under a nitrogen atmosphere at room temperature. Regardless of sample nature, PXCT derived electron density tomograms of these cylinders possessed a spatial-resolution of $\sim 50 \mathrm{~nm}$, Supporting Figure S1. Please refer to the accompanying supporting information for experimental details.

Figure 1 shows (a) volume renderings and (b) virtual cuts through the electron density tomogram of each sample. Figure 1c illustrates the corresponding electron density histograms including the theoretical electron density values of the three main catalyst components: air $\left(0.003 \mathrm{e} \AA^{-3}\right), \gamma-\mathrm{Al}_{2} \mathrm{O}_{3}\left(1.18 \mathrm{e} \AA^{-3}\right)$ and $\mathrm{MoS}_{2}\left(1.41 \mathrm{e} \AA^{-3}\right)$. See also Supporting Movie $\mathrm{S} 1$.

Examination of the bare alumina support (i) revealed the presence of two distinct matrix domains. Visible is a denser main support matrix with an average electron density of $0.365 \mathrm{e} \AA$ ${ }^{3}$ and comprising up to $\sim 50 \%$ of the whole sample volume at the given spatial-resolution (Matrix 1). Said matrix is littered with larger pores up to $3 \mu \mathrm{m}$ or greater in diameter. Located within these larger pores is a secondary minor alumina support matrix (Matrix 2) with an average electron density of $0.288 \mathrm{e}^{-3}$ and comprising up to $\sim 40 \%$ of the whole sample volume (Matrix 2). Spatially resolved pores, i.e. macropores ( $>50 \mathrm{~nm}$ ), contribute to roughly $\sim 10 \%$ of 
the sample volume, yet are highly connected even at this observation level. The five largest, identifiable, networks comprise $>60 \%$ of the resolved pore volume.

Considering the observed electron density discrepancy between pure alumina and the two matrix elements, Figure 1c and Supporting Table S1, a result of partial volume effects, we can conclude both matrix elements to possess a spatially unresolved internal porosity. Partial volume effects here refer to the occupation of a voxel by pores and alumina leading to a linear occupancy-related reduced electron density. A spatially unresolved internal porosity consisting of meso- $(2-50 \mathrm{~nm})$, and micropores $(0-2 \mathrm{~nm})$ and making up the majority of pore space in the HDS catalyst. Specifically, whereas the main support Matrix 1 possesses an internal porosity of $\sim 69 \%$, the minor Matrix element 2 owns an internal porosity of $76 \%$, i.e. is necessarily populated by more micropores and smallest mesopores. ${ }^{[10]}$ This difference in porosity is expected to be equally transcribed to differences in surface area across the matrix elements. In summary, the commercial alumina carrier possesses an overall porosity of $\sim 70$ volume per cent, well in agreement with sample-averaged physisorption (BET) measurements. According to BET measurements, present alumina carrier and resulting catalysts exhibit on average a surface area of $300 \mathrm{~m}^{2} \mathrm{~g}^{-1}$ with a pore volume $0.81 \mathrm{~cm}^{3} \mathrm{~g}^{-1}$ and an average volumetric pore diameter of $8.5 \mathrm{~nm} .^{[11]}$

The biphasic support structure is carried over into the actual HDS catalysts regardless of metal loading, Figure $1 \mathrm{~b}$ ii\&iii. Further, the size distribution of spatially resolved macropores appears to be majorly unaffected by the catalyst preparation process, hovering around $\sim 150 \mathrm{~nm}$ in diameter, Supporting Figure S2. Additionally, no voxels purely composed of molybdenum disulphide or nickel oxide could be detected at the available spatial-resolution level, in favour of a previously proposed monolayer deposition model ${ }^{[7]}$ and the major absence of large, $>(50$ $\mathrm{nm})^{3}$, molybdenum disulphide or oxide clusters. Observable is however, the expected and continued increase in electron density with progressing metal loading across both support matrices. This increase is most prominently visible in the electron density histograms shown in Figure 1c. Here the bimodal matrix distribution of the alumina carrier is replaced by a single Gaussian distribution that is progressively shifted towards higher electron densities with increasing metal loading. The continued shift of the Gaussian confirms the effective metal loading of both matrix elements. The detected change to single Gaussian distribution (ii) and the increasing peak width at full-width-half-maximum (FWHM) (iii) moreover suggests a progressively uneven metal deposition with increasing metal loading and as such active site density across the matrix elements. 
To obtain a better understanding of metal deposition across matrix elements, we isolated both matrix elements though segmentation across all tomograms (i-iii). Next, we determined the average electron density of the isolated matrices. This procedure revealed that the more porous matrix element 2 exhibits a comparatively more severe increase in electron density, i.e. a higher $\mathrm{MoS}_{2}$ particle decoration, at a metal loading of 5\% wt. Mo (ii). A behaviour that explains the observed shift to a single Gaussian distribution (Figure 1c). While this behaviour is to some degree expected, considering the potentially higher surface area of Matrix 2, unexpected is the observed reversal in electron density gains at an elevated metal loading of $10 \%$ wt. Mo and 2.3 wt. \% Ni (iii). Specifically, the electron density increase of the less porous Matrix 1 is roughly twice that of Matrix 2.

A second look at the provided cut slices, Figure $1 \mathrm{~b}$, reveals the above implied disproportionate $\mathrm{MoS}_{2}$ deposition/ formation to not occur uniform throughout Matrix 1. Visible in the sagittal cuts is a diminishing electron density gradient from the larger pore space into Matrix 1. Present gradient is suggestive of particle aggregation at the boundary of both matrix elements due to metal deposition limitations encountered upon entering the Matrix element. Selected line profiles, provided in Figure 1d, suggest this gradient to be reinforced with increasing metal loading, with selected voxel at the interface to possess an estimated $\mathrm{MoS}_{2}$ concentration of greater $20 \mathrm{wt}$. $\mathrm{MoS}_{2}$. No such gradient is observable for the minor Matrix material 2. The origin of such interfacial clustering is suggested to be a result of different pore structures. Diffusion to and deposition of Mo precursors at unique surface positions of the alumina carrier is much more likely to occur in case of the more porous matrix element 2 . We exclude Matrix element 1 undergoing coarsening, e.g. the closure of unresolved pores, during catalyst preparation associated calcination $\left(<400^{\circ} \mathrm{C}\right)$ as a secondary explanation of the detected changes in electron density. Firstly, all samples, e.g. including the bare support, were subject to higher calcination temperature $\left(>550^{\circ} \mathrm{C}\right)$ during carrier preparation, i.e. the electron density gradient should already be visible in the bare carrier. Secondly, due to the dynamic behaviour of changes in electron density, i.e. sample (ii) and (iii) have experienced the same treatment, as such should show an identical response if changes were caused by a coarsening process of the matrix.

To confirm PXCT results we examined a second nickel-containing HDS catalyst (iii) through means of focused-ion-beam milling scanning electron microscopy (FIB-SEM), scanning transmission electron microscopy (STEM) and correlated energy-dispersive X-ray 
spectroscopy (EDXS). Shown in Figure 2a is a FIB-SEM image taken from the central part of the catalyst. Visible therein is the biphasic support structure of the alumina carrier as well as the discussed density gradient into Matrix 1. See also Supporting Movie S2. Provided in Figure $2 \mathrm{~b}$ are STEM images collected of the more porous Matrix 2. Images confirm the dense but not uniform deposition of $\mathrm{MoS}_{2}$ slabs on the support, the $\mathrm{MoS}_{2}$ slab size (average thickness $>1 \mathrm{~nm}$ and length $>10 \mathrm{~nm}$ ) and most importantly the nanoporous character of the catalyst. This confirmation was sought out to justify the partial volume based analysis of PXCT tomograms. The STEM image corresponding EXDS maps are provided in Figure 2b\&c. Based on a spatial correlation of S, Mo and Ni EDXS maps it is evident that Ni deposition is decoupled from $\mathrm{MoS}_{2}$ slab location, i.e. only a fraction of $\mathrm{MoS}_{2}$ is edge-decorated with, or is in the direct vicinity of nickel-containing structures.

Lastly, to ensure that these structural observations are reflective of the entire catalyst pellet and not affected by the position of sample extraction. We prepared and analysed a second cylinder from a Ni-containing catalyst pellet (iii). Here the cylinder was extracted close to the catalyst pellet exterior, in contrast to the central position shown in Figure 1. A comparative examination of catalyst pellet core and shell showed no discernible differences on the studied length scale concerning electron density or pore size distribution, Supporting Figure S3.

In conclusion, by probing the structural make-up of an industrial alumina carrier and two HDS catalysts at increasing metal loading prepared on said carrier, we revealed a structural build of the alumina carrier consisting of two interwoven support matrix elements differing in porosity regardless of metal loading. As the studied alumina carrier and its structural derivatives, obtained through synthesis parameter adjustment, find widespread industrial utilization across different hydroprocessing applications, this observation of a biphasic build has the potential to further our understanding of a wide range of current hydroprocessing catalysts. ${ }^{[12]}$ Moreover, the observation of porosity dependent molybdenum disulphide surface coverage and aggregation at matrix element boundaries in the commercial-like catalysts will help us to understand the catalytic performance of and diffusion in current-generation HDS catalysts. While future investigations will expand on these observations examining among others the deactivation mechanism of industrially spent HDS catalysts, a more prominent effort is currently placed in the integration of X-ray spectroscopy, X-ray fluorescence and in-situ capabilities within the framework of PXCT. ${ }^{[13]}$ In consideration of next-generation synchrotron light sources, such integration will allow us on one hand to examine how differences in the 
carrier structure affect the distribution of the active phase and promoter in the catalyst. On the other, we can then also probe the chemical state and composition of the catalytically active phase during the reaction. 
References:

[1] aJ. G. Speight, in Heavy and Extra-heavy Oil Upgrading Technologies (Ed.: J. G. Speight), Gulf Professional Publishing, Boston, 2013, pp. 69-94; bJ. G. Speight, in The Refinery of the Future (Ed.: J. G. Speight), William Andrew Publishing, Boston, 2011, pp. 315-340; cH. Topsøe, B. Clausen, F. E. Massoth, Hydrotreating Catalysis, 1996.

[2] aA. N. Startsev, Catalysis Reviews 1995, 37, 353-423; bG. M. Dhar, B. N. Srinivas, M. S. Rana, M. Kumar, S. K. Maity, Catalysis Today 2003, 86, 45-60.

[3] A. Stanislaus, A. Marafi, M. S. Rana, Catalysis Today 2010, 153, 1-68.

[4] K. P. de Jong, K. P. d. Jong, Synthesis of solid catalysts, 2009.

[5] aC. Bara, A.-F. Lamic-Humblot, E. Fonda, A.-S. Gay, A.-L. Taleb, E. Devers, M. Digne, G. D. Pirngruber, X. Carrier, Journal of Catalysis 2016, 344, 591-605; bA. Villarreal, J. Ramírez, L. C. Caero, P. C. Villalón, A. Gutiérrez-Alejandre, Catalysis Today 2015, 250, 60-65.

[6] aJ. V. Lauritsen, F. Besenbacher, Journal of Catalysis 2015, 328, 49-58; bR. V. Mom, J. N. Louwen, J. W. M. Frenken, I. M. N. Groot, Nature Communications 2019, 10, 2546; cH. Topsoe, B. S. Clausen, R. Candia, C. Wivel, S. Morup, Bulletin Des Societes Chimiques Belges 1981, 90, 1189-1214; dR. Candia, O. Sørensen, J. Villadsen, N.-Y. Topsøe, B. S. Clausen, H. Topsøe, Bulletin Des Societes Chimiques Belges 1984, 93, 763-774.

[7] L. van Haandel, G. M. Bremmer, E. J. M. Hensen, T. Weber, Journal of Catalysis 2016, 342, 2739.

[8] aA. Tanimu, K. Alhooshani, Energy \& Fuels 2019, 33, 2810-2838; bH. Topsøe, B. S. Clausen, Applied Catalysis 1986, 25, 273-293.

[9] aJ. Ihli, A. Diaz, Y. Shu, M. Guizar-Sicairos, M. Holler, K. Wakonig, M. Odstrcil, T. Li, F. Krumeich, E. A. Müller Gubler, W.-C. Cheng, J. A. van Bokhoven, A. Menzel, The Journal of Physical Chemistry C 2018, 122, 22920-22929; bJ. Ihli, R. R. Jacob, M. Holler, M. GuizarSicairos, A. Diaz, J. C. da Silva, D. Ferreira Sanchez, F. Krumeich, D. Grolimund, M. Taddei, W. C. Cheng, Y. Shu, A. Menzel, J. A. van Bokhoven, Nature Communications 2017, 8, 809; cM. Holler, M. Guizar-Sicairos, E. H. R. Tsai, R. Dinapoli, E. Müller, O. Bunk, J. Raabe, G. Aeppli, Nature 2017, 543, 402-406.

[10] Y. Li, J. Su, R. Li, Microporous and Mesoporous Materials 2017, 243, 9-15.

[11] L. van Haandel, A. Longo, W. Bras, E. J. M. Hensen, T. Weber, ChemCatChem 2019, 11, 50135017.

[12] P. Robinson, Tutorial on Hydroprocessing: Hydroprocessing Catalysts and Processes, 2012.

[13] aJ. Ihli, A. Diaz, Y. Shu, M. Guizar-Sicairos, M. Holler, K. Wakonig, M. Odstrcil, T. Li, F. Krumeich, E. Müller, W.-C. Cheng, J. Anton van Bokhoven, A. Menzel, The Journal of Physical Chemistry C 2018, 122, 22920-22929; bM. Hirose, N. Ishiguro, K. Shimomura, D.-N. Nguyen, H. Matsui, H. C. Dam, M. Tada, Y. Takahashi, Communications Chemistry 2019, 2, 50; cC. Donnelly, M. Guizar-Sicairos, V. Scagnoli, M. Holler, T. Huthwelker, A. Menzel, I. Vartiainen, E. Müller, E. Kirk, S. Gliga, J. Raabe, L. J. Heyderman, Physical Review Letters 2015, 114, 115501. 
a)
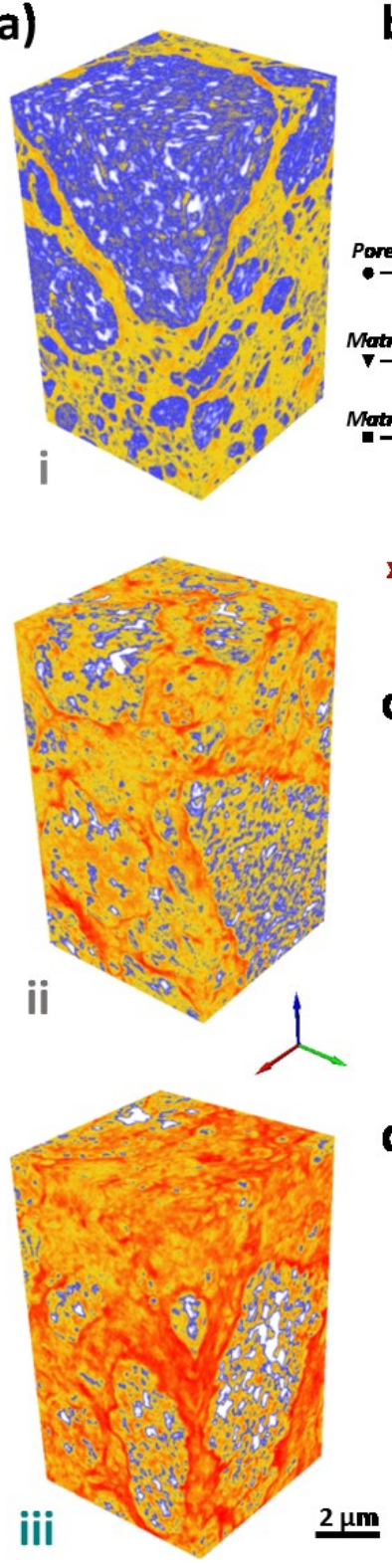
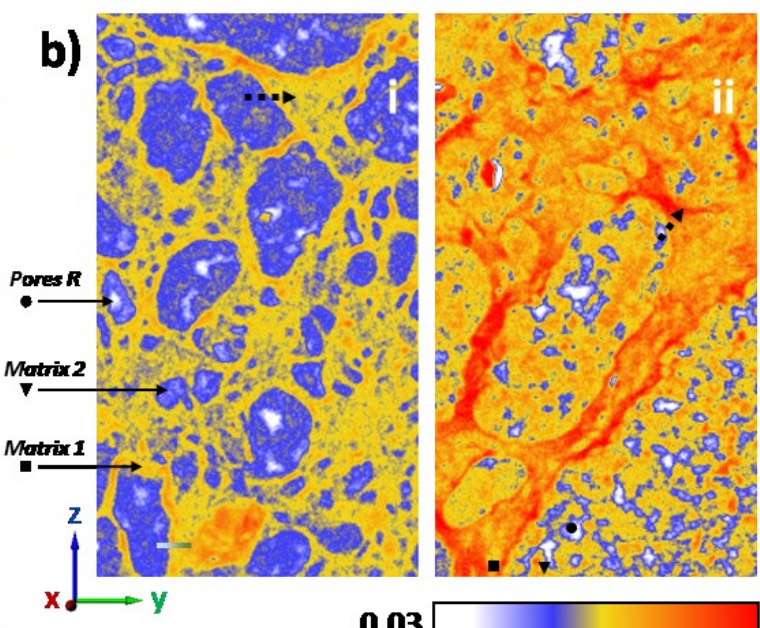

0.03

\section{Electron Density}

c)

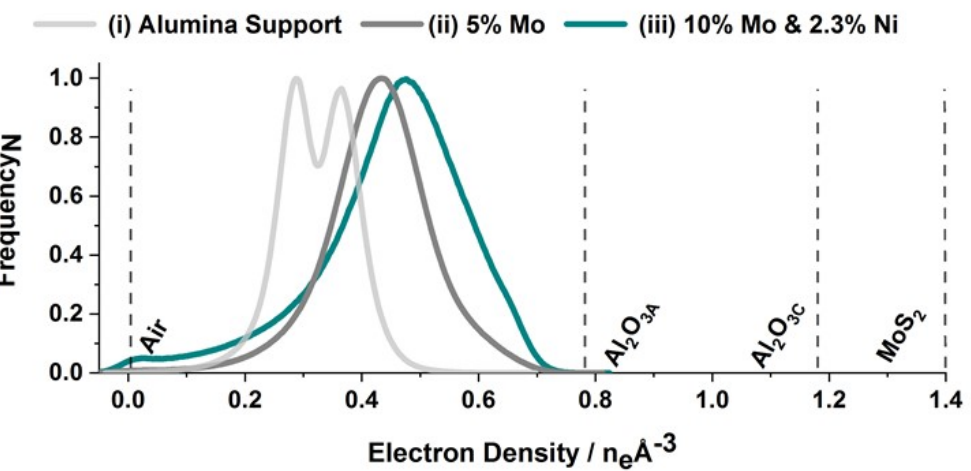

d)

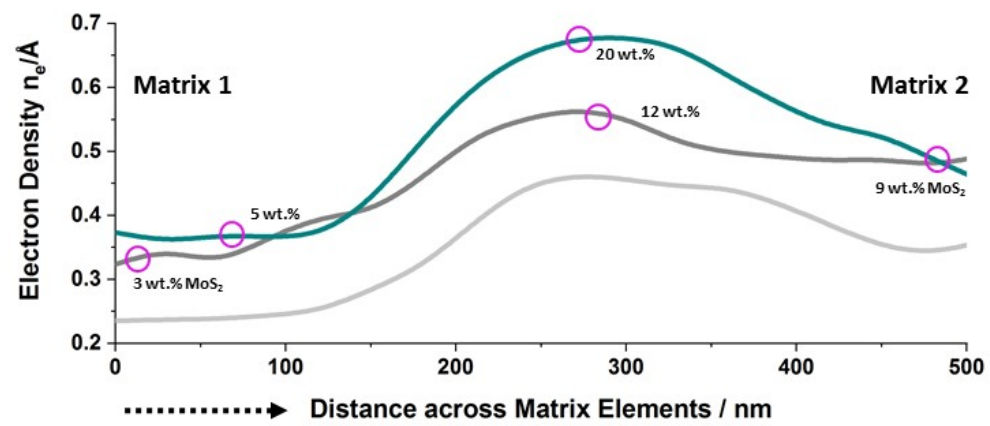

Figure 1: Ptychographic X-ray Computed Tomography of Hydrodesulphurization Catalysts. (a) Volume rendering of and (b) sagittal cuts through the electron density tomograms of HDS catalysts consisting of the (i) bare alumina support, (ii) loaded with 5 wt. \% Mo or (iii) 10 wt. \% Mo and 2.3 wt. \% Ni. Tomograms share a common colour scale ranging from white to red, where this is representative of the electron density value. Shown are isolated volumes extracted from the tomogram for comparative purposes. Major resolved pores (Pores-R $\bullet$ ) as well as meso- and more micro-porous- alumina matrix elements (Matrix 1 - \& $2 \boldsymbol{\nabla}$ ) are highlighted. The voxel size is $(30 \mathrm{~nm})^{3}$. (c) Tomogram corresponding electron density histograms. The electron densities of reference components are indicated. (d) Example electron density line profiles across the boundary from Martix element 1 into Matrix element 2 of the different HDS catalysts. The approximate position of these profiles is given by the black dashed arrows in (b). Pink circles, inferred local wt. $\mathrm{MoS}_{2}$ based on a linear combination of the average electron density of matrix elements and the theoretical electron density of $\mathrm{MoS}_{2}$. 

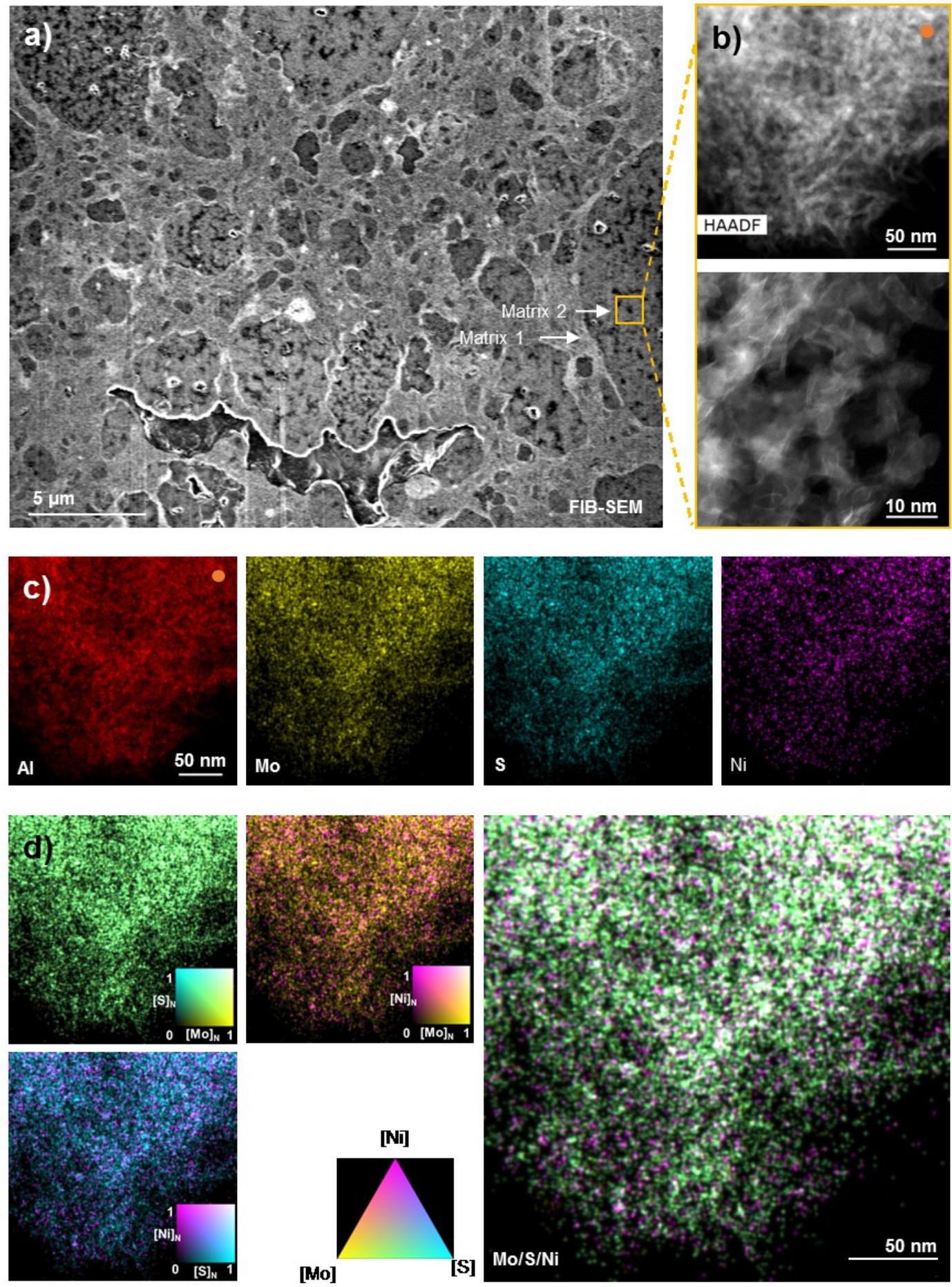

Figure 2: Electron Microscopy of a NiMo Hydrodesulphurization Catalyst. (a) FIB-SEM image collected from a nickel-enriched HDS catalyst (10 wt. \% Mo and 2.3 wt. \% Ni). (b) High-angle annular dark-field (HAADF) STEM images collected from a mechanically fractured HDS catalyst (10 wt. \% Mo and $2.3 \mathrm{wt} . \% \mathrm{Ni}$ ) at increasing magnifications. Images where acquired of Matrix 2. The orange frame is given for referencing purposes and does not indicated the area of STEM image acquisition. (c) Energy dispersive X-ray spectroscopy (EXDS) maps of aluminium (Al), sulphur (S), nickel (Ni), and molybdenum (Mo) corresponding to the STEM image shown in (b top). (d) Normalized (N) and merged EDXS maps of S, Mo and Ni, providing a visual representation of the degree of co-localization and as such an estimate of the $\mathrm{MoS}_{2}$ nickel decoration. 